\title{
Right atrial function: still underestimated in clinical cardiology
}

\author{
L. H. B. Baur
}

Received: 11 May 2008/Accepted: 13 May 2008/Published online: 30 May 2008

(C) The Author(s) 2008

As already described in several studies, the left and right atrium acts as a reservoir during ventricular systole to transport blood from, respectively, the caval veins and the pulmonary veins to the right and left ventricle, respectively. However, atrial volume and function is also associated with clinical outcome. In a multivariate risk model for the prediction of agerelated events an indexed left atrial volume above $32 \mathrm{ml} / \mathrm{m} 2$ was an independent risk factor for the prediction of the first age related cardiovascular event [1]. Although clinical outcome was corrected for age we have to realize that left atrial size and volume could be influenced by age with larger volumes found in the elderly population [2]. However, this finding has been debated in recent studies in which normal younger and elderly individuals were compared as left atrial volume is concerned [3]. Increase of left atrial volume if people get older could be related to increase of cardiac pathology at an older age: atrial fibrillation and diastolic and systolic heart failure. In contrast to the left atrium, right atrial volume and function are less well studied. This could be related to the difficulties one encounters with measuring right

L. H. B. Baur ( $ه)$

Department of Cardiology, Atrium Medical Centre

Parkstad, Henri Dunantstreet 5, 6410CX Heerlen,

The Netherlands

e-mail: 1br01@atriummc.nl

L. H. B. Baur

University of Maastricht, Maastricht, The Netherlands atrial volumes and function with $2 \mathrm{D}$ echocardiography [4]. Freehand 3D echocardiography and cardiac magnetic resonance imaging showed better results as level of agreement and intra-observer and interobserver variability is concerned [5]. Right atrial volume and function can be described by atrial filling from the point of minimal atrial volume, at the onset of ventricular systole until maximal atrial flow. It is the sum of forward caval flow and reversed flow through the tricuspid annulus. From maximal right atrial volume until atrial volume at the P-wave is described as passive emptying volume. This occurs during ventricular diastole. The active emptying volume occurs during atrial contraction until minimal atrial volume and closure of the tricuspid valve. The different phases are given in Fig. 1.

The study of Willens et al [6] in the current issue of the International Journal of Cardiac Imaging shows, that in a limited number of healthy subjects right atrial passive emptying volume and passive emptying fraction decreases with age and active emptying volume and active right atrial ejection fraction increase with age. This could indicate that with increasing age right ventricular diastolic function deceases irrespective of the occurrence of cardiac disease. In patients with pulmonary arterial hypertension right atrial function was even more impaired with larger right atrial maximal volume, minimal volume and volumes at the P-wave, lower passive right atrial emptying fraction and higher active right atrial emptying volume and active right 


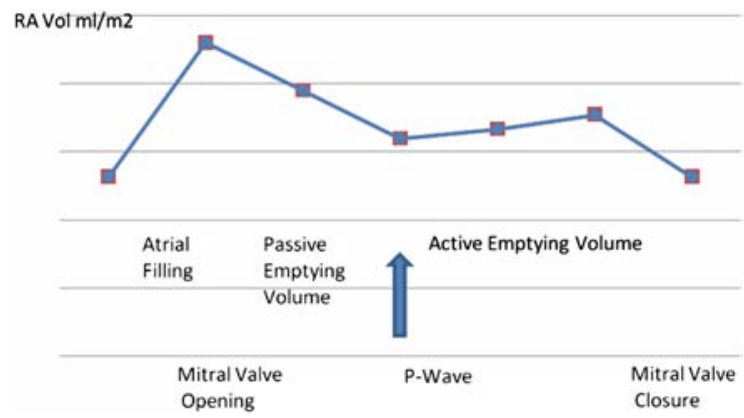

Fig. 1 Right atrial volume change over time

atrial emptying fraction pulmonary artery systolic pressure correlated fairly with active emptying volume The study of Willens gives a nice insight in right atrial mechanics and volumes in healthy individuals and patients with pulmonary hypertension. We have to take in account, that this study has several limitations. First, it is a very small study with a very limited number of patients with pulmonary hypertension included. A more serious limitation is due to the sometimes poor acoustic window of ultrasound and therefore poor definition of the right atrial endocardium and the geometric assumptions, which have been made using single plane planimetry method of discs to calculate right atrial volumes and right atrial function. As indicated, measurement of right atrial function with 3D echo or with cardiac magnetic resonance imaging in a larger cohort of normal individuals and patients could make the results more valid. The results are not without any impact. In the next decades we will see a growing number of patients with right ventricular and right atrial dysfunction: Patients with chronic pulmonary diseases, after pulmonary embolism, after right ventricular infarction, chronic heart failure, valvular heart disease and atrial fibrillation. Especially in a population with a growing number of elderly people and diseases related to higher age as atrial fibrillation and pulmonary hypertension and right ventricular dysfunction right ventricular and right atrial function has to be determined more frequently. Thus proper treatment can be initiated more accurately and the effects of treatment on the right atrium and right ventricle can be evaluated. For this, 3D echocardiography and magnetic resonance imaging are the preferred imaging tools. If these techniques are not available reasonable evaluation of right atrial function can be done with 2D echocardiography.

Open Access This article is distributed under the terms of the Creative Commons Attribution Noncommercial License which permits any noncommercial use, distribution, and reproduction in any medium, provided the original author(s) and source are credited.

\section{References}

1. Tsang TSM, Barnes ME, Gersh BJ, Takemoto Y, Rosales G, Bailey KR, Seward JB (2003) Prediction of risk for first agerelated cardiovascular events in an elderly population: the incremental value of echocardiography. J Am Coll Cardiol 42:1199-1205

2. Trisposkiadis F, Tentolouris K, Androulakis A, Trikas A, Toutouzas K, Kyriakidis M, Gialafos J, Toutouzas P (1995) Left atrial mechanical function in the healthy elderly: new insights from a combined assessment of changes in atrial volumes and transmitral flow velocity. J Am Soc Echocardiogr 8:801-809

3. Thomas L, Levett K, Boyd A, Leung DY, Schiller NB, Ross DL (2002) Compensatory changes in atrial volumes with normal aging: is atrial enlargement inevitable? J Am Coll Cardiol 40:1630-1635

4. Müller H, Burri H, Lerch R (2008) Evaluation of right atrial size in patients with atrial arrhytmias: comparison of $2 \mathrm{D}$ versus real time 3D echocardiography. Echocardiography, April 17 [Epub ahead of print]

5. Keller AM, Gopal AS, King DL (2000) Left and right atrial volume by freehand three-dimensional echocardiography: in vivo validation using magnetic resonance imaging. Eur $\mathrm{J}$ Echocardiogr 1:55-65

6. Willens HJ, Fertel DP, Qin J, Labrador E, Lowery MH (2008) Effects of age and pulmonary arterial hypertension on the different phases of right atrial function. Int $\mathrm{J}$ Cardiovasc Imaging. doi:10.1007/s10554-008-9306-4 